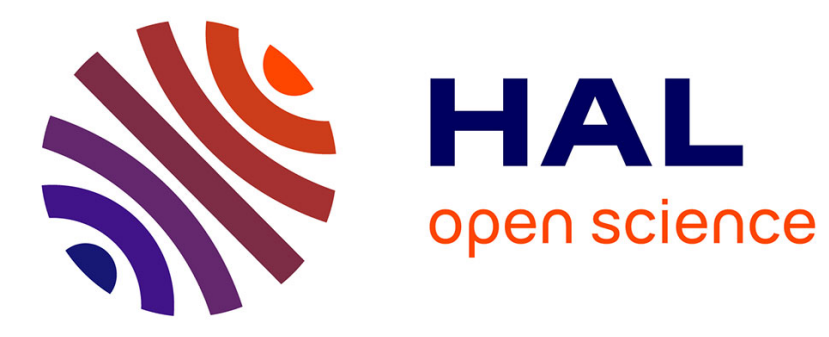

\title{
Gravity Compensation of an Upper Extremity Exoskeleton
}

Salam Moubarak, Minh Tu Pham, Richard Moreau, Tanneguy Redarce

\section{To cite this version:}

Salam Moubarak, Minh Tu Pham, Richard Moreau, Tanneguy Redarce. Gravity Compensation of an Upper Extremity Exoskeleton. 32nd IEEE EMBC, Aug 2010, Buenos Aires, Argentina. pp.4489-4493, 10.1109/IEMBS.2010.5626036 . hal-00518075

\section{HAL Id: hal-00518075 https://hal.science/hal-00518075}

Submitted on 16 Sep 2010

HAL is a multi-disciplinary open access archive for the deposit and dissemination of scientific research documents, whether they are published or not. The documents may come from teaching and research institutions in France or abroad, or from public or private research centers.
L'archive ouverte pluridisciplinaire HAL, est destinée au dépôt et à la diffusion de documents scientifiques de niveau recherche, publiés ou non, émanant des établissements d'enseignement et de recherche français ou étrangers, des laboratoires publics ou privés. 


\title{
Gravity Compensation of an Upper Extremity Exoskeleton
}

\author{
S. Moubarak, M. T. Pham, R. Moreau and T. Redarce
}

\begin{abstract}
This paper presents a new gravity compensation method for an upper extremity exoskeleton mounted on a wheel chair. This new device is dedicated to regular and efficient rehabilitation training for post-stroke and injured people without the continuous presence of a therapist. The exoskeleton is a wearable robotic device attached to the human arm. The user provides information signals to the controller by means of the force sensors around the wrist and the arm, and the robot controller generates the appropriate control signals for different training strategies and paradigms. This upper extremity exoskeleton covers four basic degrees of freedom of the shoulder and the elbow joints with three additional adaptability degrees of freedom in order to match the arm anatomy of different users. For comfortable and efficient rehabilitation, a new heuristic method have been studied and applied on our prototype in order to calculate the gravity compensation model without the need to identify the mass parameters. It is based on the geometric model of the robot and accurate torque measurements of the prototype's actuators in a set of specifically chosen joint positions. The weight effect has been successfully compensated so that the user can move his arm freely while wearing the exoskeleton without feeling its mass.
\end{abstract}

Keywords- Upper extremity exoskeleton, gravity compensation, rehabilitation, medical robotics.

\section{INTRODUCTION}

Stroke and accident victims, hemiplegic subjects and spinal cord injured persons suffer from many forms of handicaps for locomotion because of the decline of their physical and muscular strength. Robotic rehabilitative training has recently become more common, and thus, commercial robotic devices are already available and increasingly needed to facilitate and accelerate the training and the recovery of weak and injured people.

For the upper limbs, several studies have been carried out and most researchers have paid increasing attention to develop upper extremity exoskeletons for the medical training and rehabilitation [15]-[19].

Manuscript received April 23, 2010. This work was supported by EC project MEST-CT-2005-021024 WARTHE.

S. Moubarak is with the Institut National des Sciences Appliquées (INSA) de Lyon, 69621 Villeurbanne Cedex, France (phone: 0033-4-72-4362-13; fax: 0033-4-72-43-85-35; e-mail: salam.moubarak@insa-lyon.fr).

M. T. Pham is with the Institut National des Sciences Appliquées (INSA) de Lyon, 69621 Villeurbanne Cedex, France (e-mail: minh-tu.pham@insa-lyon.fr).

R. Moreau is with the Institut National des Sciences Appliquées (INSA) de Lyon, 69621 Villeurbanne Cedex, France

(e-mail: richard.moreau@insa-lyon.fr).

T. Redarce is with the Institut National des Sciences Appliquées (INSA) de Lyon, 69621 Villeurbanne Cedex, France

(e-mail: tanneguy.redarce@insa-lyon.fr).
This paper deals with the development and the gravity compensation of an upper extremity exoskeleton mounted on a wheel chair (Fig. 1). Our objectives are to provide an efficient rehabilitation and physiotherapy for the patient's arm, as well as the possibility for the therapist to apply different training strategies with a variable assistance degree depending on the state of the user and the injury level. Furthermore, this device enables the patient to have a consistent training without the continuous assistance of the therapist; therefore, the latter can work on many patients simultaneously and the total cost of the therapy can be reduced.

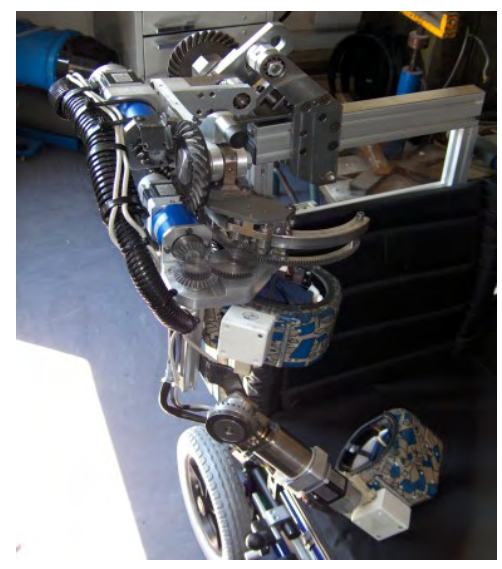

Fig. 1. The upper extremity exoskeleton

An important criterion for transparent handling and comfortable rehabilitation with exoskeletons is the efficient gravity compensation in order to achieve a compliant controller so that the user can move his arm freely without feeling the weight of the robot [7].

For anthropomorphic-sized arms it has been shown that even for fast motions, the use of a PD controller with gravity compensation apparently performs almost as well as a full feedforward controller that accommodates full inertial terms $[1,2]$ and most researchers accorded a great importance for the development of accurate gravity compensation models for serial rigid robots [7, 8], elastic robots [3] and parallel mechanisms [9]. In [1], identification protocols have been carried out in order to estimate the mass parameters of robots for gravity compensation applications. In [4], a mechanical gravity canceller based on passive compensation mechanisms has been studied in order to reduce the gravity effects on the manipulators' links.

Our work introduces a new method for the identification of the gravity term of the exoskeleton without knowing the 
value of its mass parameters. Our objective is to calculate an accurate assessment of the dynamic model's gravity component in order to eliminate the weight effect by providing the necessary and sufficient actuator torques to hold the robotic arm in any given stance of its workspace without blocking it.

The paper is organized as follows: Section 2 describes the different components of our prototype. Section 3 presents our new gravity compensation method. Finally, a brief conclusion highlights the ongoing works and the future works of this project.

\section{A NEW UPPER EXTREMITY EXOSKELETON}

The prototype has 4 motorized dofs; the shoulder abduction / adduction, flexion / extension and internal / external rotation as well as the elbow flexion / extension motion (Fig. 2 - 3). The shoulder height, the shoulder width and the arm length can be adapted to fit with different body sizes for different users. Each motor is connected to a drive and can be controlled either by torque or by velocity. The 4 drives and the adaptability motors are connected to a dSPACE control board and continuously exchanging information and control signals. Different control schemes can be created and applied using Matlab / Simulink and dSPACE -ControlDesk interface.

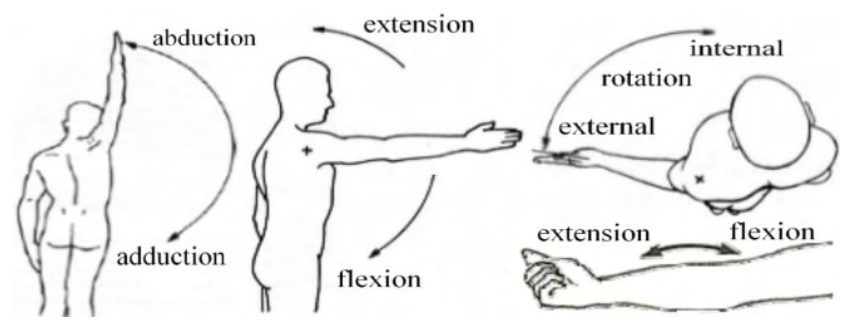

Fig. 2. The four basic degrees of freedom of the arm

\section{II.1- Mechanical structure:}

The main purpose of an exoskeleton is not only to provide efficient motion assistance to the human limbs, but also to guarantee the safety and the comfort of the user. That is why matching the human body anatomy is one of the most important criteria for an exoskeleton design.

The mechanical structure of our prototype (Fig. 3) mainly consists of four links and four revolute joints covering the basic degrees of freedom of the human arm. The motion range of the robot's joints is limited to provide a wide but risk-free workspace for the user. The arm is attached to the exoskeleton by external arm and wrist holders with pressure adjustable internal pneumatic holders. The rotation motion is transmitted from the motor axes to the joint axes by rigid and compact spiro-conical gear systems (Fig. 4). The prototype is relatively lightweight $(10 \mathrm{~kg})$ with a high ratio of dofs / weight.

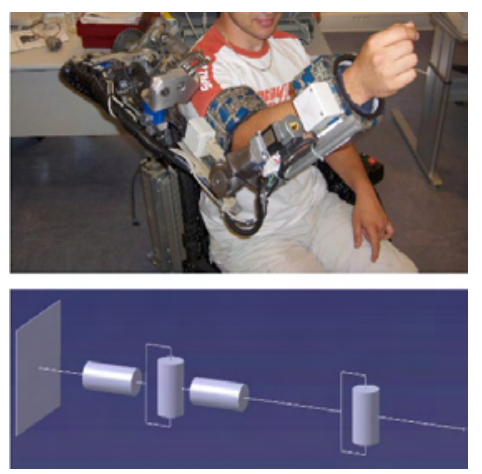

Fig. 3. The mechanical structure of the exoskeleton

\section{II.2- Actuators:}

The four active dofs of the prototype are motorized by brushless motors with high torque and relatively small dimensions. The motor torques are amplified by the reducers and the gear systems, to give final output torques of $63 \mathrm{Nm}$ for the shoulder abduction / adduction, $52.5 \mathrm{Nm}$ for the shoulder flexion / extension, $17.1 \mathrm{Nm}$ for the shoulder internal / external rotation, and $13.5 \mathrm{Nm}$ for the elbow flexion / extension motion.
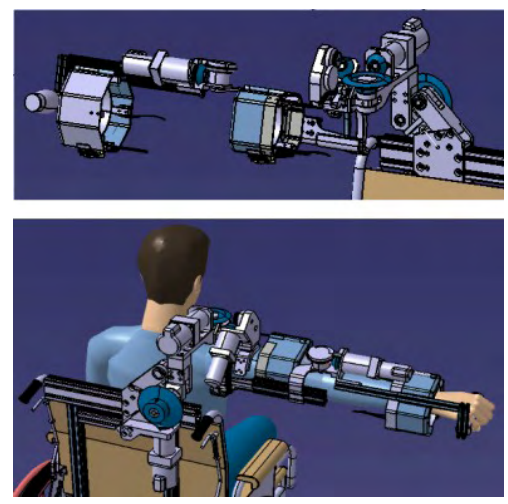

Fig. 4. The gear systems and the arm holders of the exoskeleton

The prototype must be adaptable so it can be used by different patients with different body sizes. The shoulder height, the shoulder width and the arm length can be varied using three DC motors to fit with the users' body dimensions.

\section{II.3- Control system:}

All brushless motors are equipped with high resolution magnetic encoders for position feedback measurements. Each motor is connected to a drive with a sampling frequency of $5 \mathrm{kHz}$. The 4 drives and the 3 adaptability DC motors are connected to the same electronic interface board.

The electronic board exchanges information and control signals with a dSPACE controller analyzing all the feedback information and sending the appropriate control signals to the system depending on the training strategy 


\section{GRAVITY COMPENSATION OF THE EXOSKELETON}

III.1- The gravity term of the dynamic model:

The inverse dynamic model of a robot calculates the required joint torques as a function of the joint positions, velocities and accelerations. It can be written [5]:

$$
\Gamma=A(q) \ddot{q}+C(q, \dot{q}) \dot{q}+Q(q)+F_{D} \operatorname{sign}(\dot{q})+F_{V} \dot{q}(1)
$$

$A$ is the inertia matrix of the robot, $C$ is the matrix containing the centrifugal and Coriolis torques, $Q$ is the gravity torques vector, $F_{D}$ and $F_{V}$ respectively represent the dry friction and viscous friction terms, $\Gamma$ is the actuator torque; $q, \dot{q}, \ddot{q}$ respectively represent the joints position, velocity and acceleration vectors.

Theoretically, for any given position $q, Q(q)$ is the torque vector that we should apply to the robot's joints in order to support the total weight of the mechanical structure without succeeding it. Therefore, it can be maintained suspended in its position without being blocked, enabling the user to move the robot freely, without lifting its weight.

Full dynamic parameter identification methods are wellknown in robotics $[10,11,12]$. In these methods, the dynamic parameters are estimated using the least squares solution of a linear system obtained from the sampling of the dynamic model along a closed loop tracking trajectory. The main difficulty lies in the derivation estimation to obtain velocity and acceleration. Without any precaution, this process induces noise on the identified parameter. Furthermore, these algorithms require persisting excitation signals in order to proceed to the identification. Finally, these approaches tend to complicate the identification process if the only need is to find out the gravity term.

Our original gravity compensation method leads to the assessment of the gravity term based on the geometric model and a finite set of torque measurements appropriately chosen. Contrary to other methods, this technique does not need any identification protocols of the mass parameters.

\section{III.2- The geometric modeling:}

The geometric modeling and notations are based on the Modified Denavit-Hartenberg (MDH) notation [13]: We assume the serial robot has $\mathrm{n}+1$ links denoted $\left(L_{0}, \ldots L_{j}, \ldots L_{n}\right)$ and $\mathrm{n}$ joints $(1, \ldots j, \ldots n)$. The joint $j$ connects the link $j$ with the link $j-1$. The link 0 is the base of the robot

The joint variable $j$ is denoted $q_{j}$. For such a description, a frame is attached to each link $j$ using an iterative and systematic procedure [13]:

The $z_{j}$ axis is along the axis of joint $j$. The $x_{j}$ axis is along the common normal between $z_{j}$ and $z_{j+1}$. If the axes $z_{j}$ and $z_{j+1}$ are parallel, the choice of $x_{j}$ is not unique.

The origin $O_{j}$ is the intersection of $x_{j}$ and $z_{j}$.

The geometric modelling of the robot is based on the four geometric parameters:

$\alpha_{j}$ : angle between $z_{j-1}$ and $z_{j}$ around $x_{j-1}$

$d_{j}$ : distance between $z_{j-1}$ and $z_{j}$ along $x_{j-1}$

$\theta_{j}$ : angle between $x_{j-1}$ and $x_{j}$ around $z_{j}$

$r_{j}$ : distance between $x_{j-1}$ and $x_{j}$ along $z_{j}$

The joint variable $q_{j}$ is equal to $\theta_{j}$ for a revolute joint and $r_{j}$ for a prismatic joint.

\section{III.3- The new gravity compensation method:}

For each joint $j$, we use the transformation matrix between the base frame and the frame $j$ to calculate the gravity vector $\vec{g}$ in the frame $j:\left(\vec{g}_{j}\right)$, and project it along $x_{j}, y_{j}$ and $z_{j}$ to give the algebraic values $g_{x j}, g_{y j}$ and $g_{z j}$.

For any given position of the robot, the gravity compensation torque that should be applied on the joint $j$

$\left(\Gamma_{j}\right)$ is equal to the opposite sum of the torques applied around $z_{j}$ by the links $\left(L_{j}, \ldots L_{n}\right)$ under the effect of $\vec{g}_{x j}$, $\vec{g}_{y j}$ and $\vec{g}_{z j}\left(\Gamma_{x j}, \Gamma_{y j}\right.$ and $\left.\Gamma_{z j}\right)$ :

$\Gamma_{j}=-\left(\Gamma_{x j}+\Gamma_{y j}+\Gamma_{z j}\right)$

$\vec{g}_{z j}$ being collinear to $z_{j}$, it has no effect on the torque around $z_{j}$, thus $\Gamma_{z j}=0$ and equation (2) can be written:

$$
\Gamma_{j}=-\left(\Gamma_{x j}+\Gamma_{y j}\right)
$$

Fig. 5 shows the kinematic structure of the exoskeleton in the initial position. In this position, the frames 0 and 1 are superposed.

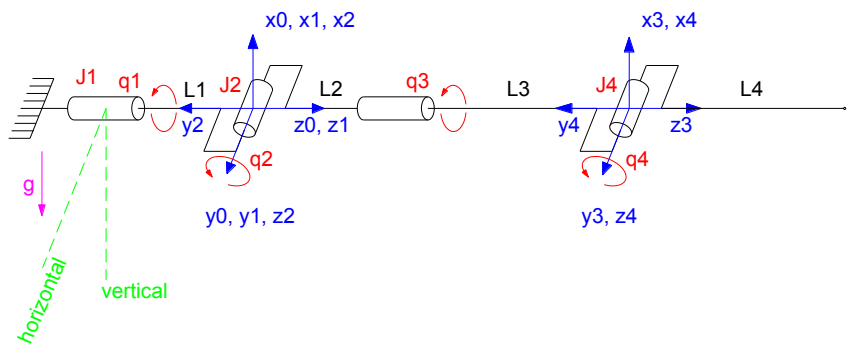

Fig. 5. The kinematic structure of the prototype

The gravity compensation torques are calculated by a 
heuristic method, based on several static torque measurements in a set of appropriately chosen joint positions as well as a thorough geometric analysis of the robot's structure. Let us define:

$C_{1}$ : measured torque on $j_{4}$ for $q=\left[\begin{array}{llll}0 & 0 & 0 & 0\end{array}\right]^{T}$

$C_{2}$ : measured torque on $j_{4}$ for $q=\left[\begin{array}{llll}0 & -\frac{\pi}{2} & 0 & 0\end{array}\right]^{T}$

$C_{3}$ : measured torque on $j_{3}$ for $q=\left[\begin{array}{llll}0 & 0 & 0 & 0\end{array}\right]^{T}$

$C_{4}$ : measured torque on $j_{3}$ for $q=\left[\begin{array}{llll}0 & 0 & -\frac{\pi}{2} & 0\end{array}\right]^{T}$

$C_{5}$ : measured torque on $j_{2}$ for $q=\left[\begin{array}{llll}0 & 0 & 0 & 0\end{array}\right]^{T}$

$C_{6}$ : measured torque on $j_{2}$ for $q=\left[\begin{array}{llll}0 & -\frac{\pi}{2} & 0 & 0\end{array}\right]^{T}$

$C_{7}$ : measured torque on $j_{1}$ for $q=\left[\begin{array}{llll}0 & 0 & 0 & 0\end{array}\right]^{T}$

$C_{8}$ : measured torque on $j_{1}$ for $q=\left[\begin{array}{cccc}-\frac{\pi}{2} & 0 & 0 & 0\end{array}\right]^{T}$

A careful and methodical geometric analysis of the structure leads to the following equations:

$$
\begin{aligned}
& \Gamma_{x 4}=\frac{g_{x 4}}{g} C_{1} \\
& \Gamma_{y 4}=\frac{g_{y 4}}{g} C_{2} \\
& \Gamma_{x 3}=\frac{g_{x 3}}{g} C_{3} \\
& \Gamma_{y 3}=\frac{g_{y 3}}{g}\left(C_{4}-C_{2}+C_{2} \cos \left(q_{4}\right)+C_{1} \sin \left(q_{4}\right)\right) \\
& \Gamma_{x 2}=\frac{g_{x 2}}{g}\left(C_{5}-C_{1}+C_{1} \cos \left(q_{4}\right)-C_{2} \sin \left(q_{4}\right)\right) \\
& \Gamma_{y 2}=\frac{g_{y 2}}{g}\left(\begin{array}{l}
C_{6}-C_{4}+C_{3} \sin \left(q_{3}\right) \\
+\left(C_{4}-C_{2}\right) \cos \left(q_{3}\right) \\
+C_{2} \cos \left(q_{4}\right) \cos \left(q_{3}\right) \\
+C_{1} \sin \left(q_{4}\right) \cos \left(q_{3}\right)
\end{array}\right) \\
& \Gamma_{x 1}=\frac{g_{x 1}}{g}\left(\begin{array}{l}
C_{7}-C_{3}+C_{3} \cos \left(q_{3}\right) \\
-\left(C_{4}-C_{2}\right) \sin \left(q_{3}\right) \\
-C_{2} \cos \left(q_{4}\right) \sin \left(q_{3}\right) \\
-C_{1} \sin \left(q_{4}\right) \sin \left(q_{3}\right)
\end{array}\right)
\end{aligned}
$$

$$
\Gamma_{y 1}=\frac{g_{y 1}}{g}\left(\begin{array}{l}
C_{8}-C_{6}+\left(C_{6}-C_{4}\right) \cos \left(q_{2}\right) \\
+\left(C_{5}-C_{1}\right) \sin \left(q_{2}\right) \\
+\left(C_{4}-C_{2}\right) \cos \left(q_{3}\right) \cos \left(q_{2}\right) \\
+C_{3} \sin \left(q_{3}\right) \cos \left(q_{2}\right) \\
+C_{1} \cos \left(q_{4}\right) \sin \left(q_{2}\right) \\
+C_{1} \sin \left(q_{4}\right) \cos \left(q_{3}\right) \cos \left(q_{2}\right) \\
-C_{2} \sin \left(q_{2}\right) \sin \left(q_{4}\right) \\
+C_{2} \cos \left(q_{2}\right) \cos \left(q_{3}\right) \cos \left(q_{4}\right)
\end{array}\right)
$$

Based on equation (3) and equations (4...11), we can apply the torque $\Gamma_{j}$ on each joint $j$ in order to totally compensate the gravity effect on the exoskeleton. Note that $\left(g_{x 1 \ldots 4}\right)$ and $\left(g_{y 1 \ldots 4}\right)$ can be calculated in terms of $\left(q_{1 \ldots 4}\right)$ based on the geometric model of the robot.

\section{III.4- Theoretical validation:}

The general form of the $j^{\text {th }}$ element of the gravity term vector $Q(q)$ for an n-dof rigid robot arm is given by [14]:

$$
Q_{j}(q)=-\sum_{k=j}^{n} m_{k} \widetilde{g}^{T} \frac{\partial T_{0}^{k}}{\partial q_{j}} \bar{r}_{k}^{k}
$$

Where, in terms of homogeneous transform description, $\tilde{g}=\left[\bar{g}^{T}, 0\right]^{T}=[-g, 0,0,0]^{T}$ (for our prototype), and $\bar{r}_{j}^{j}=\left[\left(\tilde{r}_{j}^{j}\right)^{T}, 1\right]^{T}=\left[\widetilde{r}_{x j}^{j}, \tilde{r}_{y j}^{j}, \widetilde{r}_{z j}^{j}, 1\right]^{T}$ is the mass center vector of the link $L_{j}$ with respect to the origin of the frame $\mathrm{j}$.

In order to theoretically validate our gravity compensation model, the constants $\left(C_{1} \ldots C_{8}\right)$ were symbolically calculated as a function of the mass parameters of the robot and substituted by their symbolic expressions in equations (4...11). Then, based on equation 3, the gravity compensation torque of each joint was calculated and compared to equation (12). The results showed full equality between the 2 forms; thus, our gravity compensation method was theoretically validated.

\section{III.5- Experimental validation:}

An experimental validation was carried out by applying our gravity compensation model and positioning the prototype in a series of static postures. The gravity effect proved to be successfully compensated, for the robot could be left suspended in any position of its workspace without moving or dropping under its weight effect.

Note that it is possible, depending on the training paradigm and the state of the user, to compensate not only the weight of the robot, but also the user's arm weight. The 
only difference would be in the torque measurements $\left(C_{1} \ldots C_{8}\right)$; they would have to be made with the users arm resting in the exoskeleton.

\section{CONCLUSION AND FURTHER WORKS}

This paper presents a new upper extremity exoskeleton mounted on a wheel chair and dedicated to the training and rehabilitation of injured people and accident victims as well as post-stroke patients in order to improve and accelerate the recovery process.

A new, exact and simple method for the gravity compensation of the exoskeleton has been developed in order to improve the prototype's comfort and performance as well as its potential for more efficient therapy and training modes. It is based on:

- the geometric model of the robot

- accurate torque measurements of the prototype's actuators in a set of specifically chosen joint positions.

It has several advantages compared to other solutions, especially the fact that it can accurately identify the gravity term of the dynamic model without the need to identify the value of the prototype's mass parameters.

We have shown in this paper an experimental validation of the compensation technique and a theoretical validation through a comparison between the gravity term obtained with our method and the theoretical gravity term of the dynamic model.

Another aspect of this work concerns the control strategy. In the future works, the gravity compensation will be applied and force sensors mounted around the wrist and the arm will be used to estimate the state and the motion intentions of the user and create efficient training and therapy strategies for a better and faster recovery.

\section{REFERENCES}

[1] D. Ma, J. M. Hollerbach, "Identifying Mass parameters for gravity compensation and Automatic Torque Sensor Calibration", Proceedings of the 1996 IEEE International Conference on Robotics and Automation, Minneapolis, Minnesota, pp. 661 - 666, April 1996,

[2] C. H. An, C. G. Atkenson, J. M. Hollerbach, Mode- based control of a Robot Manipulator. Cambridge, MA: MIT Press, 1988

[3] L. Zollo, A. De Luca, B. Siciliano, "Regulation with on-line gravity compensation for robots with elastic joints", Proceedings of the 2004 IEEE International Conference on Robotics and Automation, New Orleans, LA. pp. 2687 - 2692, April 2004

[4] T. Morita, F. Kuribara, Y Shiozawa, S. Sugano, "A Novel Mechanism Design for Gravity Compensation in Three Dimensional Space", Proceedings of the 2003 IEEE/ASME International Conference on advanced intelligent Mechatronics (AIM 2003), pp. $163-168$

[5] W. Khalil and E. Dombre, Modeling, Identification and Control of Robots, Elsevier Butterworth Heinemann, 1999

[6] R. A. Cooper, B. E. Dicianno, B. Brewer, E. LoPresti, D. Ding, R. Simpson, G. Grindle, H. Wang. "A perspective on intelligent devices and environments in medical rehabilitation, Medical Engineering \& Physics", Volume 30, Issue 10, pp 1387-1398, December 2008.
[7] R. Ott, M. Gutirrez, D. Thalmann, F. Vexo, "Improving user comfort in haptic virtual environments through gravity compensation", Eurohaptics Conference, pp 401- 409, 2005

[8] M. Liu and N. H. Quach. "Estimation and compensation of gravity and friction forces for robot arms: Theory and experiments", International Journal of Intelligent Robotics System, 31(4):339-354, 2001

[9] D. Checcacci, E. Sotgiu, A. Frisoli, C. Avizzano, and M. Bergamasco. "Gravity compensation algorithms for parallel haptic interface", Proceedings of IEEE International Workshop on Robot and Human Interactive Communication, Berlin, Germany, pages 140-145, 2002.

[10] C. H. An, C. G. Atkenson, and J. M. Hollerbach, "Estimation of inertial parameters of rigid body links of manipulators" Proceedings of the Conference on Decision and Control, pages 990-995, 1985.

[11] M. Gautier "Dynamic identification of robots with power model", Proceedings of IEEE International Conference on Robotics and Automation, pages 1922-1927, Albuquerque, USA, 1997

[12] P.K. Khosla, Real-time control and identification of direct drive manipulators. Ph. D. Thesis, Carnegie Mellon University, 1986.

[13] W. Khalil and J.-F. Kleinfinger, "A new geometric notation for open and closed-loop robots", Proceedings of IEEE International Conference on Robotics and Automation, pages 1174-1180, San Francisco, CA USA, 1986

[14] Paul, R. P., Robot manipulators : Mathematics, Programmind, and Control, MIT Press, Cambridge, MA, 1983

[15] E. Rocon, A.F. Ruiz, F. Brunetti, and J.L. Pons, "On the use of an active wearable exoskeleton for tremor suppression via biomechanical loading", IEEE International conference on robotics and automation, Orlando, Florida, Mai 2006, pp.3140-3145.

[16] K. Kiguchi, Y. Imada, and M. Lianage, "EMG-Based Neuro-Fuzzy Control of a 4DOF power-assist exoskeleton", 29th IEEE EMBS International conference, Cité internationale, Lyon, France, August 2007, pp.3040-3043.

[17] R. Song, K.Y. Tong, X.L. Hu, S.F. Tsang, and L. Li, "The therapeutic effects of myoelectrically controlled robotic system for persons after stroke-A pilot study ", 28th IEEE EMBS International conference, New York city, USA, August-September 2006, pp.3504-3511.

[18] M. Mihelj, T. Nef, and R. Reiner, "ARMin II-7DOF rehabilitation robot: mechanics and kinematics", IEEE International conference on robotics and automation, Romania, Italy, April 2007, pp.4120-4125.

[19] A. Denève, "Développement et commande d'un robot pour la rééducation des membres supérieurs", Ph.D. dissertation, University of Reims Champagne-Ardenne, France, 2007.

[20] Khalil, W., \& Creusot, D. (1997). SYMORO+: A system for the symbolic modelling of robots. Robotica, 15, 153-161. 